Z. Epileptol. 2021 · 34:27-35

https://doi.org/10.1007/s10309-020-00378-w

Angenommen: 2. Dezember 2020

Online publiziert: 29. Dezember 2020

(c) Der/die Autor(en) 2020

\section{Definition und anatomischer Überblick}

Im Gegensatz zu Frontal- oder Temporallappenepilepsien ist die Definition von Epilepsien, die von den hinteren Hirnanteilen ausgehen, weniger präzise. Dies ist zum Teil darauf zurückzuführen, dass Anfälle aus diesen Hirnregionen seltener eine typische Semiologie zeigen und weniger einfach zu lokalisieren sind. Die epileptogene Zone ist im posterioren Kortex weniger häufig auf einen Lappen begrenzt, sodass Resektionen häufig über diese Grenzen hinaus erfolgen. Aus diesem Grund werden Epilepsien, die vom Partietal-, Okzipital- und posterioren Temporallappen ausgehen, oft als Epilepsien des posterioren Kortex zusammengefasst [1]. Nach derzeitigem Wissensstand geht man davon aus, dass Epilepsien des posterioren Kortex weniger als $10 \%$ aller refraktären Epilepsien ausmachen. In der Gruppe der Patienten, die sich einer präoperativen Diagnostik unterziehen, schwankt der Prozentsatz der Epilepsien des posterioren Kortex und des Okzipitallappens zwischen 2 und $20 \%$, wobei Kinder häufiger betroffen sind als Erwachsene [1-3].

Epilepsien des posterioren Kortex stellen für Epileptologen eine besondere Herausforderung dar, da erste klinische Anzeichen eines Anfalls häufig erst nach einer Propagation in andere Hirnareale auftreten. Anfälle ahmen daher typische

\author{
Stephan Steinhoff' · Julia Jacobs ${ }^{2,3,4}$ \\ ${ }^{1}$ Klinik für Neurologie, Kliniken der Stadt Köln, Köln-Merheim, Deutschland \\ ${ }^{2}$ Abteilung für Pädiatrische Neurologie und Muskelkrankheiten, Medizinisches Zentrum - Universität \\ Freiburg, Medizinische Fakultät, Universität Freiburg, Freiburg, Deutschland \\ ${ }^{3}$ Department of Paediatrics and Department of Neuroscience, Cumming School of Medicine, University of \\ Calgary, Calgary, Kanada \\ ${ }^{4}$ Hotchkiss Brain Institute and Alberta Children's Hospital Research Institute, University of Calgary, Calgary, \\ Kanada
}

\title{
Mehr Propagation als Lokalisation: Anfälle aus dem posterioren Kortex
}

Frontal- oder Temporallappensemiologie nach. Ein detailliertes Wissen über die Propagationswege und Vernetzung posteriorer Hirnareale ist daher notwendig, um zu verstehen, welche Symptome Hinweise auf eine Epilepsie mit posteriorem Ursprung sein können. Dieser Review fasst die aktuelle Literatur über Propagationswege und Netzwerkstrukturen zusammen. Die meisten aktuellen Erkenntnisse hierzu kommen aus Studien, die systematisch iktale Propagation mit intrakraniellen Elektroden untersuchen.

\section{Klassifikation von Epilepsien des posterioren Kortex}

In der aktuellen Literatur finden sich prinzipiell 2 unterschiedliche Klassifikationen für Epilepsien des posterioren Kortex. Zum einen werden unterschiedliche Formern danach klassifiziert, welche anatomischen Strukturen in die Anfallsgenerierung involviert sind bzw. welche Strukturen operiert werden müssen, um Anfallsfreiheit $\mathrm{zu}$ erreichen. Die meisten epilepsiechirurgischen Serien unterscheiden zwischen isolierter Okzipitallappenepilepsie (OLE), isolierter Parietallappenepilepsie und kombinierten Typen wie Okzipitoparietallappen-, Okzipitotemporallappen- und Okzipitoparietotemporallappenepilepsie, immer abhängig von der Größe der Operation, die durchgeführt wird [4-6]. Auch wenn die Nomenklatur variabel ist, besteht das
Schlüsselprinzip dieser Studien darin zu untersuchen, bei welchem Prozentsatz der Patienten epileptogene Areale die Lappengrenzen überschreiten und ob sich dies positiv oder negativ auf die postoperative Anfallsfreiheit auswirkt. Je nach Studie beschränkten sich zwischen 12 und $35 \%$ der Operationen auf nur einen Lappen/eine Lokalisation, während multilobäre Resektionen insbesondere in der Pädiatrie häufiger vorkamen $[3,5,6]$.

Eine zweite Möglichkeit zur Klassifizierung von posterioren Epilepsien ist deren Klassifikation auf der Basis typischer Propagationswege. Craciun et al. unterscheiden hierbei zwischen 2 Propagationswegen: zum einen eine ventrale Propagation von posterior nach temporal/mesiotemporal und zum anderen eine dorsale Propagation von posterior nach frontal und parietal [7]. Diese Differenzierung ist v. a. hilfreich, um iktale Semiologien $\mathrm{zu}$ verstehen und $\mathrm{zu}$ unterscheiden. Typische motorische Symptome des Frontallappens traten in der Gruppe mit dorsaler Ausbreitung signifikant häufiger auf, während typische Temporallappensymptome wie orale und manuelle Automatismen fast ausschließlich in der Gruppe mit ventraler Ausbreitung auftraten. Diese Einteilung ist aber nicht exklusiv und die alleinige Beurteilung der Anfallssemiologie oft nicht ausreichend um Propagationswege komplett zu verstehen. 


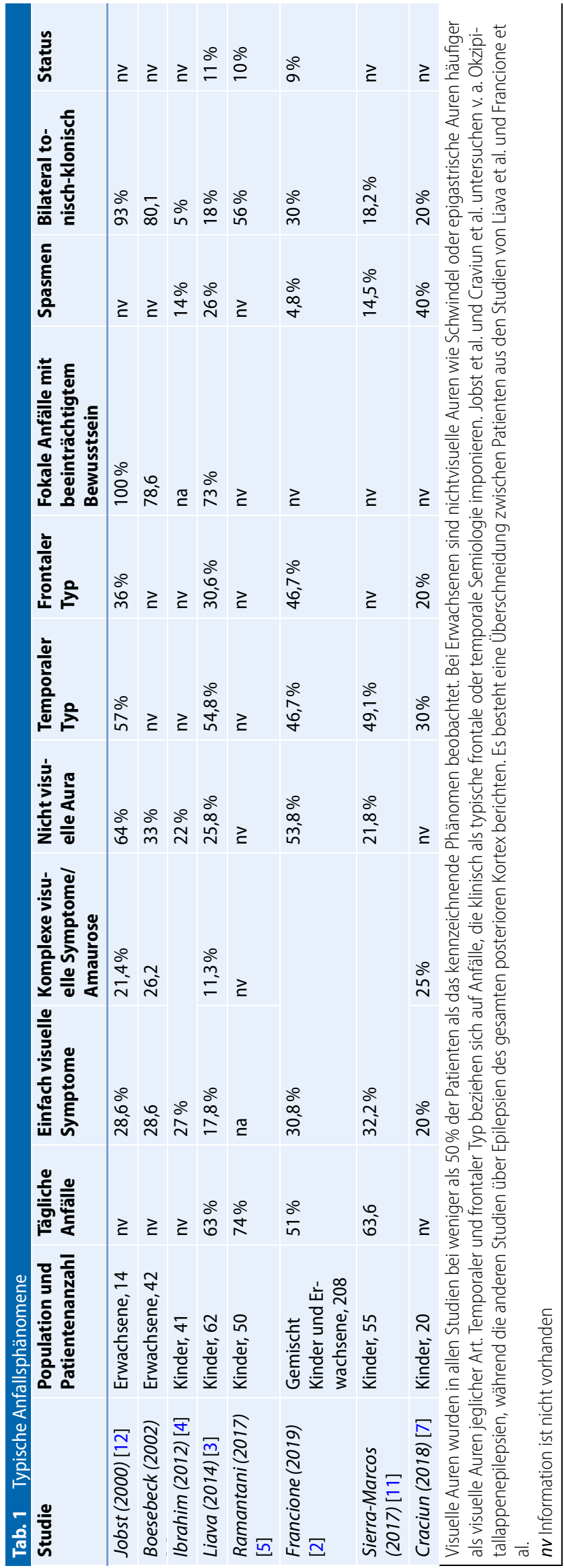

Zusammenfassend sind bei posterioren Epilepsien häufig weite Netzwerke in die Generierung und Ausbreitung von epileptischen Anfällen involviert. Prognose und Operationserfolg sind wie bei allen fokalen Epilepsien besser, wenn die präoperative Diagnostik einen lokalisierten Anfallsursprung zeigt.

\section{Anatomische Überlegungen und funktionelle Zusammen- hänge}

Der parietookzipitale Sulcus trennt den Okzipitallappen auf der mesialen Oberfläche vom Temporal- und Parietallappen. Die calcarine Fissur trennt mesial den Cuneus (O6) vom Gyrus lingualis (O5). Vor dem Gyrus lingualis liegt der Gyrus parahippocampalis. Aufderlateralen Oberfläche des Okzipitallappens liegen der obere, mittlere und untere okzipitale Gyrus jeweils untereinander und verschmelzen nach posterior laufend zum Okzipitalpol $[8,9]$. Der primäre visuelle Kortex, eine für die Epilepsiechirurgie wichtige Struktur, liegt im mesialen Aspekt des Okzipitallappens um den calcarinen Sulcus herum. Der primäre visuelle Kortex ist über den Tractus geniculocalcarinus mit dem Corpus geniculatum laterale verbunden. Zusätzliche Faserverbindungen verbinden die oberen Netzhautfelder mit dem oberen Teil des Sulcus calcarinus. Die Fasern des unteren Netzhautfeldes durchlaufen im Gegensatz dazu den Temporallappen, bevor sie das primäre Gesichtsfeld erreichen. Während die Kenntnis der Fasern aus den primären Gesichtsfeldern für die Epilepsiechirurgie essenziell ist, um einen Funktionsverlust zu vermeiden, scheinen sie für die Anfallspropagation weniger relevant zu sein. Stattdessen scheint Anfallspropagation hauptsächlich über schnelle Faserverbindungen stattzufinden, die den primären visuellen Kortex mit den visuellen Assoziationsfeldern verbinden.

Die visuellen Assoziationskortizes befinden sich sowohl im lateralen und oberen Bereich des Okzipitallappens als auch in den hinteren Teilbereichen. Sie umfassen viele Aufgaben, die die visuelle Funktion unterstützen, wie das Erkennen, Interpretieren und Wahrnehmen von Farbe, Tiefe und Bewegung. Lange 
Assoziationsbündel verbinden diese $\mathrm{Re}$ gionen mit präfrontalen, motorischen, insularen, sensorischen und temporalen Arealen [8]. Frontallappen und visuelle Assoziationsbereiche sind direkt über die superioren longitudinalen und inferioren frontookzipitalen Faszikel verbunden [10]. Darüber hinaus sind primäre und sekundäre visuelle Kortexareale über interhemisphärische Verbindungen miteinander verbunden. Letzteres ist die Erklärung für die oft falsch lateralisierenden oder weitverbreiteten Oberflächen-EEG-Befunde bei Okzipitallappenepilepsien [3, 5].

Wie oben beschrieben, gehen die Gyri des Okzipitallappens mesial direkt in posteriore temporale Areale über. Die parietookzipitale Fissur bildet eine sichtbare Grenze zwischen Okzipital- und Parietallappen auf der mesialen Oberfläche. Lateral hingegen ist der obere Parietallappen (P1) direkt mit dem Gyrus occipitalis superior verbunden. In ähnlicher Weise geht der Gyrus angularis (P2) in den mittleren Gyrus occipitalis über, und der untere Gyrus temporalis geht direkt in den unteren Gyrus occipitalis über, wobei ihre Trennung nur durch die Incisura praeoccipitalis sichtbar wird. Diese Kontinuität erklärt, warum so viele Läsionen nicht auf einen Lappen beschränkt sind, sondern den hinteren Quadranten umfassen.

Funktionell gesehen, ist der Gyrus postcentralis, der primäre sensorische Funktionen erfüllt, der am besten verstandene Teil des Parietallappens. Wie der Okzipitallappen besitzt der Parietallappen lange Assoziationsbündel, die die somatisch-sensorischen Bereiche mit dem frontalen und visuellen Assoziationskortex [10] verbinden. Der sensorische Kortex ist über die subkortikale extrapyramidale Bahn ebenfalls eng mit den primären motorischen Arealen verbunden. Darüber hinaus verbindet das Corpus callosum die sensorischen Areale der beiden Hemisphären direkt miteinander.

Die Funktionen des superioren $\mathrm{Pa}$ rietallappens sind weniger gut verstanden, und Anfälle aus dieser Region zeigen keine typisch bekannte Anfallssemiologie. Der superiore Parietallappen beherbergt sensorische Assoziationsbereiche

Z. Epileptol. 2021·34:27-35 https://doi.org/10.1007/s10309-020-00378-w

(c) Der/die Autor(en) 2020

\section{S. Steinhoff · J. Jacobs}

\section{Mehr Propagation als Lokalisation: Anfälle aus dem posterioren Kortex}

\section{Zusammenfassung}

Epilepsien mit einem Anfallsursprung aus dem Okzipital-, Parietal- und posterioren Temporallappen werden häufig als Epilepsien des posterioren Kortex zusammengefasst. Gemeinsam haben diese Epilepsien, dass symptomatogene Zone und Anfallsursprung oft nicht oder nur teilweise überlappen. Anfälle aus dem posterioren Kortex können klinisch wie Temporal- oder Frontallappenanfälle imponieren. Diese Übersichtsarbeit diskutiert klinische Merkmale und typische

Präsentationen dieser Epilepsien. Ein detailliertes Wissen über Netzwerkverbindung zwischen den okzipitalen und parietalen Strukturen und anderen Hirnregionen ist für eine erfolgreiche chirurgische Behandlung dieser Epilepsien unerlässlich.

Schlüsselwörter

Posteriorer Kortex - Epilepsie · Anfallssemiologie $\cdot$ Netzwerke $\cdot$ Epilepsiechirurgie

\section{More propagation than localization: seizures from the posterior cortex}

\begin{abstract}
Epilepsies with seizures deriving from the occipital, parietal and posterior temporal lobes are frequently grouped together as epilepsies of the posterior cortex. These epilepsies have in common that the symptomatogenic zone and origin of the seizure often do not overlap or only partially overlap. Seizures from the posterior cortex can clinically have the same appearance as temporal and frontal lobe seizures. This review article discusses the
\end{abstract}

clinical features and typical presentation of these epilepsies. A profound understanding of network connections between the occipital and parietal structures and other brain regions is essential for successful surgical treatment of these epilepsies.

Keywords

Posterior cortex · Epilepsy · Seizure semiology . Networks · Epilepsy surgery und hat viele direkte Verbindungen mit dem ipsilateralen Cingulum, dem Gyrus temporalis superior, dem Gyrus postcentralis und der okzipitalen Region. Dies ermöglicht eine rasche Ausbreitung der iktalen Aktivität, und die meisten hier erzeugten Anfälle sind höchstwahrscheinlich erst dann symptomatisch, wenn eine iktale Propagation stattgefunden hat.

Es bleibt umstritten, ob die Insel ein häufig involviertes Areal bei Epilepsien des posterioren Kortex ist. Einige Autoren untersuchen die hintere Insel im Rahmen intrakranieller Implantationen [3]. Eine mögliche Propagation in die posteriore Insel ist nicht unwahrscheinlich da der Fasziculus arcuatus an die dorsale Insel und der untere Fasziculus occipitofrontale an die untere Insel grenzt.

\section{Klinische Besonderheiten bei posterioren Epilepsien}

\section{Ätiologie}

Die Ätiologie der refraktären posterioren Epilepsien unterscheidet sich wenig von anderen fokalen refraktären Epilepsien. Viele der diagnostizierten Läsionen (45-68\%) hatten im MRT unklare Grenzen und überschritten die Lappengrenzen $[2,11]$. Letzteres könnte erklären, wieso $60 \%$ der Erwachsene ein intrakranielles EEG erhielten, obwohl eine eindeutige strukturelle Veränderung im MRT sichtbar war [2].

\section{Anfallssemiologie}

Die Epilepsie des posterioren Kortex ist das Chamäleon unter den Epilepsien, da die Anfallssemiologie stark variiert 


\begin{tabular}{|c|c|c|c|c|c|c|c|c|c|}
\hline Studie & $\begin{array}{l}\text { Interiktal, } \\
\text { lokalisie- } \\
\text { rend }\end{array}$ & $\begin{array}{l}\text { Interiktal, } \\
\text { laterali- } \\
\text { sierend }\end{array}$ & $\begin{array}{l}\text { Interiktal, falsch } \\
\text { lokalisierend/ } \\
\text { lateralisierend }\end{array}$ & $\begin{array}{l}\text { Iktal, } \\
\text { lokali- } \\
\text { sierend }\end{array}$ & $\begin{array}{l}\text { Iktal, } \\
\text { laterali- } \\
\text { sierend }\end{array}$ & $\begin{array}{l}\text { Iktal, falsch lo- } \\
\text { kalisierend/ } \\
\text { lateralisierend }\end{array}$ & $\begin{array}{l}\text { \% der } \\
\text { intrakrani- } \\
\text { ellen EEGs }\end{array}$ & $\begin{array}{l}\text { Interiktales } \\
\text { iEEG, lokali- } \\
\text { sierend }\end{array}$ & $\begin{array}{l}\text { Iktales } \\
\text { iEEG, loka- } \\
\text { lisierend }\end{array}$ \\
\hline $\begin{array}{l}\text { Jobst (2000) } \\
{[12]}\end{array}$ & $43 \%$ & $93 \%$ & $7 \%$ & $43 \%$ & $93 \%$ & $7 \%$ & $100 \%$ & nv & nv \\
\hline $\begin{array}{l}\text { Boesebeck } \\
\text { (2002) [6] }\end{array}$ & $66 \%$ & $29 \%$ & $5 \%$ & nv & $59,5 \%$ & $10 \%$ & $0 \%$ & nv & nv \\
\hline $\begin{array}{l}\text { Ibrahim }^{\text {b }} \\
\text { (2012) [4] }\end{array}$ & $71 \%$ & $12 \%$ & $21 \%$ & $76 \%$ & $4 \%$ & $20 \%$ & $56,1 \%$ & nv & nv \\
\hline $\begin{array}{l}\text { Liava (2013) } \\
\text { [3] }\end{array}$ & $35,5 \%$ & $98 \%$ & $19,3 \%$ (lok) & 37,5 & $32 \%$ & $25 \%$ & $38,7 \%$ & $46 \%$ & $80 \%$ \\
\hline $\begin{array}{l}\text { Ramantani } \\
\text { (2017) [5] }\end{array}$ & $34 \%$ & $66 \%$ & & $54 \%$ & $46 \%$ & & $26 \%$ & nv & nv \\
\hline $\begin{array}{l}\text { Francione } \\
\text { (2019) [2] }\end{array}$ & $\begin{array}{l}16 \% \\
(40 \%)^{a}\end{array}$ & $18 \%$ & $11 \%$ & $\begin{array}{l}28 \% \\
(50 \%)^{a}\end{array}$ & $9 \%$ & $5,5 \%$ & 55,8 & nv & nv \\
\hline $\begin{array}{l}\text { Sierra- } \\
\text { Marcos } \\
\text { (2017) [11] }\end{array}$ & nv & $\mathrm{nv}$ & $20 \%$ & $63 \%$ & $83,3 \%$ & $16,7 \%$ & 36,4 & nv & $\mathrm{nv}$ \\
\hline $\begin{array}{l}\text { Craciun } \\
\text { (2018) [7] }\end{array}$ & nv & nv & nv & $20 \%$ & $80 \%$ & $20 \%$ & $100 \%$ & nv & nv \\
\hline \multicolumn{10}{|c|}{$\begin{array}{l}\text { Die Autoren Jobst und Liava zählen alle lokalisierenden Befunde auch als lateralisierende Befunde, während die anderen Publikationen EEGs entweder als } \\
\text { lateralisierend oder lokalisierend klassifizieren. Ramantani et al. differenzieren nur zwischen regionalen vs. nichtregionalen EEG-Veränderungen. Francione et al } \\
\text { unterscheiden zwischen lokalisierend und regional ('Zahl in Klammern bezieht sich auf regional). Bei Ibrahim et al. wird die EEG-Korrelation nur für anfallsfreie } \\
\text { Patienten angegeben ( }{ }^{b} 27 \text { Patienten) } \\
n v \text { Information nicht verfügbar }\end{array}$} \\
\hline
\end{tabular}

und Semiologien aller anderen Hirnareale nachahmen können. Hierfür gibt es 2 Hauptgründe: erstens, dass viele Hirnareale im posterioren Kortex keine objektiv sichtbaren positiven Anfallsphänomene hervorrufen; zweitens, die ausgedehnte Vernetzung der hinteren Gehirnareale mit anderen Hirnregionen über schnelle Bahnstrukturen. Während bestimmte lokalisierende Phänomene wie einfache visuelle Auren und okulärmotorische Zeichen als klarer Hinweis in Richtung des Okzipitallappens gewertet werden sollten, sind andere Auren wie Schwindel weniger spezifisch. Darüber hinaus treten bei vielen Patienten nur Anfallsphänomene, die normalerweise nicht dem posterioren Kortex zugeschrieben werden, auf. Selbst bei bestätigter Okzipitallappenepilepsie werden visuelle Phänomene nur bei etwas mehr als $50 \%$ der Erwachsenen berichtet [12]. In - Tab. 1 werden die Anfallsphänomene einiger größerer Studien zusammengefasst. Viele Symptome können auf eine Beteiligung des posterioren Kortex hindeuten, sind jedoch selten spezifisch genug, um eine chirurgische Planung ohne weitere Untersuchungen $\mathrm{zu}$ ermöglichen. Einige Studien schlagen eine Unterscheidung von typischen frontalen und temporalen Anfallsphänomenen vor, und daher werden diese in • Tab. 1 separat aufgeführt [7]. Als typische temporale Phänomene wurden hierbei folgende Symptome gewertet: manuelle und orale Automatismen, Bewusstseinsverlust sowie epigastrische oder Déjà-vuAuren. Frontale Phänomene waren in der Regel motorische Manifestationen wie tonische Körperhaltung, kontralaterale Kloni und hypermotorische Symptomatik $[3,7,11]$.

Die starke Vernetzung des posterioren Kortex könnte auch einige andere Phänomene erklären, die studienübergreifend beschrieben werden. Die Mehrheit der Patienten hat einen Anfallsbeginn in sehr jungem Alter, wobei das Durchschnittsalter selbst in den Serien der erwachsenen Patienten bei etwa 6 Jahren liegt [2, 5]. Bei Patienten mit multilobärem Anfallsursprung war das Durchschnittsalter zu Epilepsiebeginn mit 1 bis 2 Lebensjahren sogar noch niedriger. Ein relativ großer Anteil der Patienten weist epi- leptische Spasmen auf, bis zu 50\% der Patienten mit „Okzipital-Plus-Epilepsie“ [3]; 8-12\% der Patienten zeigen epileptische Spasmen über das erste Jahr hinaus und bis zur Operation [4, 7, 11]. Darüber haben viele Patienten eine ungewöhnlich hohe Anfallsfrequenz, und die Hälfte der Erwachsenen hat täglich Anfälle [2]; $10-30 \%$ der Patienten erleiden mindestens einen Status epilepticus.

\section{Typische Ergebnisse der präoperativen Diagnostik}

Ähnlich wie die Anfallssemiologie sind auch die interiktalen und iktalen EEGBefunde alles andere als eindeutig und einheitlich. Selten treten unilaterale fokale epilepsietypische Potenziale (ETP) über einer Region auf, meist zeigen sich multifokale, bilaterale oder sogar falsch lokalisierende ETPs. Während fokale unilaterale ETPs als prognostisch relevant für ein anfallsfreies Operationsergebnis angesehen werden, bedeutet ein weit ausgedehntes Feld von ETPs nicht unbedingt das Gegenteil. Die - Tab. 2 fasst Erkenntnisse über die Lokalisation 


\begin{tabular}{|c|c|c|c|c|c|c|c|c|c|}
\hline Studie & $\begin{array}{l}\% \text { an- } \\
\text { falls- } \\
\text { frei }\end{array}$ & $\begin{array}{l}\text { Alter bei } \\
\text { Epilepsie- } \\
\text { beginn }\end{array}$ & $\begin{array}{l}\text { Dauer } \\
\text { der Epi- } \\
\text { lepsie }\end{array}$ & Ätiologie & $\begin{array}{l}\text { Ober- } \\
\text { flächen- } \\
\text { EEG }\end{array}$ & $\begin{array}{l}\text { Intra- } \\
\text { krani- } \\
\text { elles } \\
\text { EEG }\end{array}$ & $\begin{array}{l}\text { Resektions- } \\
\text { größe/ } \\
\text { Vollständig- } \\
\text { keit }\end{array}$ & $\begin{array}{l}\text { Lokalisation } \\
\text { der Resekti- } \\
\text { on }\end{array}$ & Anderes \\
\hline $\begin{array}{l}\text { Jobst (2000) } \\
{[12]}\end{array}$ & $50 \%$ & nv & nv & Nein & nv & nv & Nein & $\mathrm{Ja}$ & - \\
\hline $\begin{array}{l}\text { Boesebeck } \\
\text { (2002) [6] }\end{array}$ & 45 & nv & nv & $\begin{array}{l}\text { Läsionell } \\
\text { Tumor }>\text { MCD }\end{array}$ & $\begin{array}{l}\text { Nein (nur } \\
\text { postope- } \\
\text { rativ) }\end{array}$ & $\mathrm{nv}$ & Nein & Nein & $\begin{array}{l}\text { Lateralisierende Aura > nicht } \\
\text { lateralisierende Aura }\end{array}$ \\
\hline $\begin{array}{l}\text { Ibrahim } \\
\text { (2012) [4] }\end{array}$ & $68 \%^{\mathrm{a}}$ & Nein & Ja & Nein & Nein & nv & Nein & Nein & $\begin{array}{l}\text { Kontralateraler MEG Dipol als } \\
\text { negative Prognose }\end{array}$ \\
\hline $\begin{array}{l}\text { Liava (2013) } \\
{[3]}\end{array}$ & $85,5 \%$ & Ja & Nein & $\begin{array}{l}\text { Strukturell > } \\
\text { nicht läsionell }\end{array}$ & $\mathrm{nv}$ & $\mathrm{Ja}$ & $\mathrm{Ja}$ & Nein & SEEG prognostisch negativ \\
\hline $\begin{array}{l}\text { Ramantani } \\
\text { (2017) [5] }\end{array}$ & $70 \%$ & Nein & Ja & Nein & Nein & $\mathrm{nv}$ & Nein & $\begin{array}{l}\text { Parietal }< \\
\text { okzipital } \\
L<R \text { Hemis- } \\
\text { phäre }\end{array}$ & - \\
\hline $\begin{array}{l}\text { Francione } \\
\text { (2019) [2] }\end{array}$ & $69,7 \%$ & nv & $\mathrm{nv}$ & Nein & nv & Ja & nv & Ja & $\begin{array}{l}\text { SEEG mit negativer Prognose } \\
\text { bezüglich des Outcomes }\end{array}$ \\
\hline $\begin{array}{l}\text { Sierra- } \\
\text { Marcos } \\
(2017)[11]\end{array}$ & $62 \%$ & Nein & nv & Strukturell & $\begin{array}{l}\text { Post- } \\
\text { operativ } \\
\text { IED }\end{array}$ & nv & nv & Nein & $\begin{array}{l}\text { Nummer der Antikonvulsiva } \\
\text { und hohe Anfallsfrequenz } \\
\text { prognostisch schlecht }\end{array}$ \\
\hline $\begin{array}{l}\text { Craciun } \\
(2018)[7]\end{array}$ & $68,7 \%$ & Nein & $\mathrm{nv}$ & Strukturell & nv & nv & nv & Ja & $\begin{array}{l}\text { Patienten mit frontaler Propa- } \\
\text { gation > temporale Propaga- } \\
\text { tion, Hypometabolismus im } \\
\text { PET mit negativer Prognose }\end{array}$ \\
\hline
\end{tabular}

von Oberflächen-EEG-Befunden und ihre prognostische Relevanz aus einigen neueren Studien zusammen. Nicht nur die interiktalen, auch iktale EEG-Muster können falsch lokalisieren [3, 7]. Vor allem wenn die erste iktale EEG-Aktivität erst spät innerhalb des klinischen Anfalls auftritt, sollte man bei der Interpretation des EEGs vorsichtig sein. Dies trifft natürlich auf alle Epilepsieformen $\mathrm{zu}$, ist aber für Epilepsien mit posteriorem Ursprung besonders häufig beschrieben.

Positive MRT-Befunde sind wahrscheinlich der häufigste Grund für die Überlegung, dass sich die Anfallsursprungszone im posterioren Kortex befinden könnte. Weitere beschriebene Warnhinweise sind ein Hypometabolismus im FDG-PET im posterioren Kortex. Vor allem bei nichtläsionalen Temporallappenepilepsien sollte ein posterior zum Temporallappen gelegener Hypometabolismus immer weitere Untersuchungen nach sich ziehen, um sicherzustellen, dass keine Okzipitallappenepilepsie übersehen wird [7].
Neuropsychologische Untersuchungen werden häufig in der präoperativen Diagnostik verwendet, um eine Lokalisationshypothese $\mathrm{zu}$ bestätigen oder Zweifel an ihr auszuräumen. Dies ist bei Epilepsien aus dem posterioren Kortex besonders kompliziert. Erstens sind viele Parietallappenfunktionen schwierig $\mathrm{zu}$ testen und eher unspezifisch [13]. Zweitens haben viele Patienten mit frühem Beginn und häufigen Anfällen eine insgesamt niedrige kognitive Leistungsfähigkeit und können nicht formell getestet werden [11]. Das spezifischste fokale Defizit ist ein vorbestehender Gesichtsfelddefekt, der bei 10-36\% der Patienten auftritt und häufig strukturelle Veränderungen im Okzipitalbereich als Ursache hat $[3,5,7]$.

\section{Postoperative Anfallsfreiheit}

Frühe Berichte über Epilepsien des posterioren Kortex sind hinsichtlich des postoperativen Anfallsergebnisses eher entmutigend. Vor allem auch aufgrund des hohen Risikos für visuelle Funktionsdefizite, die durch die Operation verursacht werden. Boesebeck et al. haben epilepsiechirurgische Studien bis zum Jahr 2000 zusammengefasst. Hierbei zeigte sich eine hohe Varianz mit Raten für die Anfallsfreiheit nach Operation zwischen 25 und $90 \%$ [6]. In der Mehrheit der Studien lag die Anfallsfreiheitsrate jedoch um und unter $50 \%$ [1, 14-16].

Von den 208 Patienten aus der italienischen Studie von Francione wurden $70 \%$ nach der Operation anfallsfrei [2]. Interessanterweise war dies in der mit intrakraniellem EEG untersuchten Gruppe seltener der Fall als in der Gruppe ohne diese Untersuchung (80 vs. $61 \%$ ). Vermutlich liegt dies an der höheren Komplexität der Fälle in der zweiten Gruppe. Kürzlich wurden für Kinder vergleichbar gute Ergebnisse publiziert, 65-85\% der Patienten waren nach der Operation anfallsfrei [3, 5, 11]. Dies ist besonders vielversprechend angesichts der Schwere der Erkrankung und ihres häufig frühen Beginns. Die - Tab. 3 fasst die Ergebnis- 


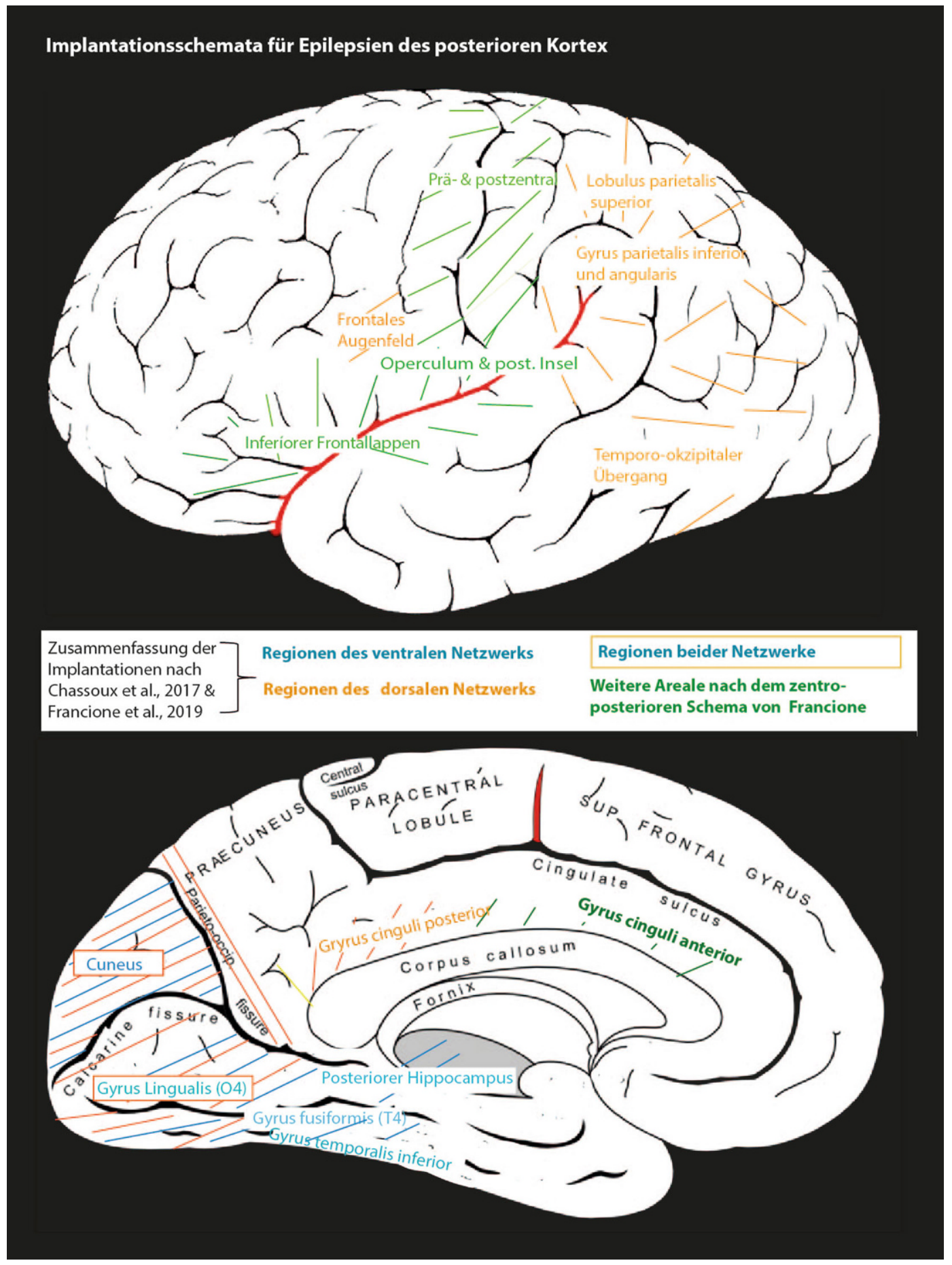

Abb. $1 \varangle$ Fallbeispiel einer 6 Jahre alten Patientin mit 2 epileptogenen Läsionen. Anfallssemiologie mit beeinträchtigtem Bewusstsein, Augenwendung nach links, vereinzelt mit iktalem Erbrechen. Intrakranielle Elektroden wurden in folgenden Hirnregionen platziert: Amygdala, anteriorer und posteriorer Hippocampus (rote Markierung), anteriore und posteriore Insel, zentrales und parietales Operculum, im Gyrus parietalis superior und 3 okzipitale Elektroden: infra-, supracalcarin und Pol (gelbe Markierung). Anfälle mit Ursprung in der Läsion supracalcarin (oberes $E E G$ ), iktales Erbrechen konnte klar auf die Propagation ventral zum Hippocampus hin zurückgeführt werden (unteres EEG). Unabhängig elektrographische Anfälle aus dem Hippocampus. Läsionektomie okzipital und selektive Amygdalahippokampektomie nach intrakraniellem EEG. Patientin ist seit 2 Jahren anfallsfrei

se und Einflussfaktoren der verschiedenen operativen Studien zusammen. Zusammenfassend lässt sich sagen, dass die untersuchten Faktoren nicht einheitlich sind und einige Studien zu widersprüchlichen Ergebnissen kommen. Der Einfluss des Alters bei Epilepsiebeginn, der Dauer der Epilepsie, der Ätiologie und der Lokalisation der Resektion auf die postoperative Anfallsfreiheit bleibt daher un- klar. Den Einfluss der Ätiologie auf das Operationsergebnis zu kommentieren ist auch deshalb schwierig, weil viele Studien nur Patienten mit struktureller Epilepsie einschließen.

\section{Besonderheiten bei Kindern}

Wie bereits erwähnt, ist der Epilepsiebeginn häufig im Kindesalter. Die ho- he Anzahl der Anfälle und die Epilepsieschwere erfordern häufig eine frühe operative Therapie. Als mögliche Ursache für den frühen Beginn wird die Verteilung der physiologischen Hirnreifung bei Kindern diskutiert. Der posteriore Kortex ist einer der ersten Teile des Gehirns, der eine Differenzierung und Reifung der grau-weißen Substanz zeigt und den größten Teil seiner Schlüsselfunk- 


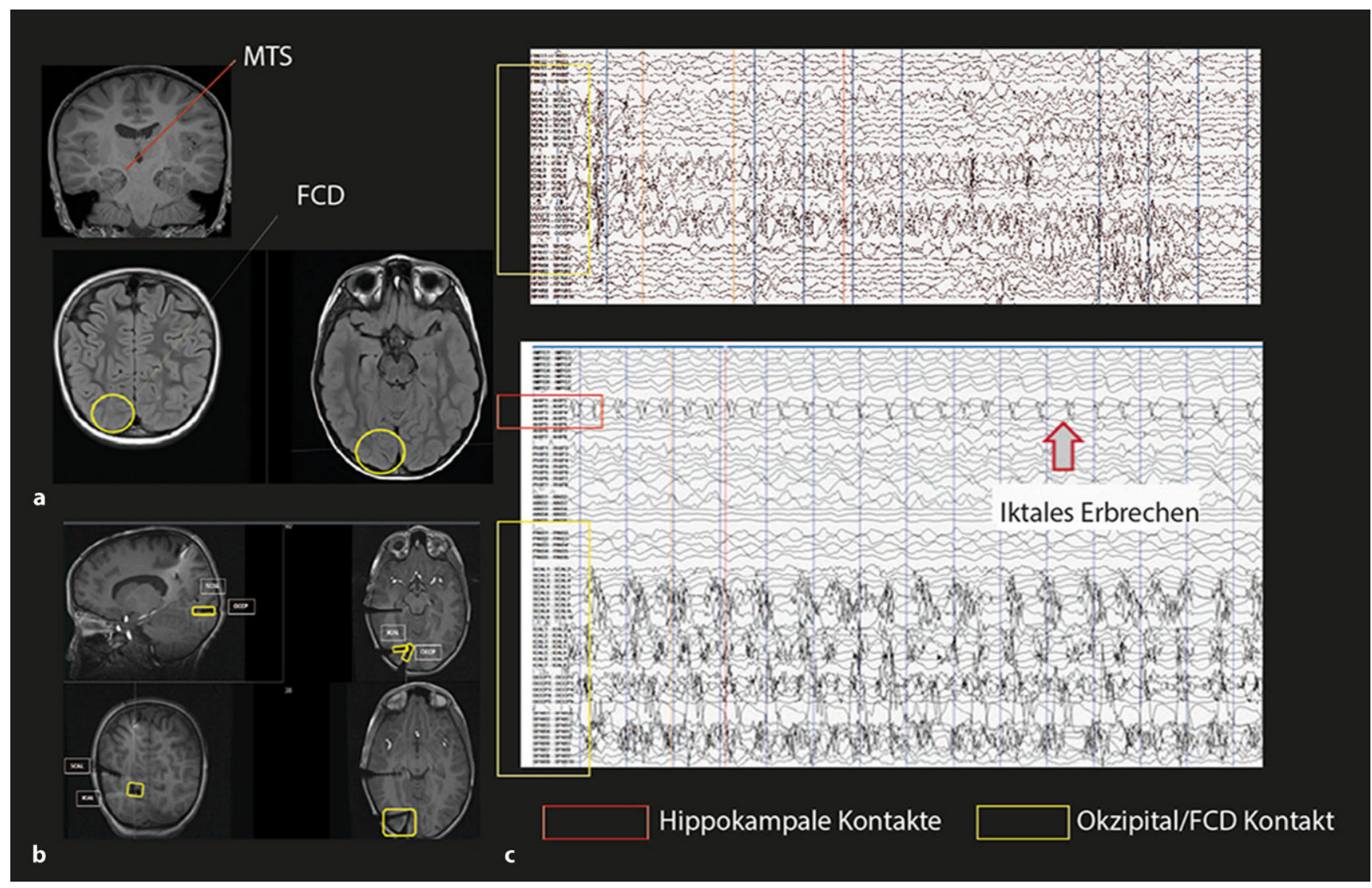

Abb. 2 ム Übersicht der verschiedenen empfohlenen Implantationschemata bei Epilepsien des posterioren Kortex. Ziel ist es, verschiedene Propagationswege akkurat zu verstehen

tionen innerhalb der ersten Lebensjahre entwickelt $[17,18]$. Im Fall von posterioren Läsionen wird diese Reifung verzögert, und dies kann sich frühzeitig auf die gesamte Netzwerkstruktur des Gehirns auswirken.

Eine Herausforderung bei der Behandlung von Kindern ist ihre altersbedingte Unfähigkeit, über subjektive Anfallsphänomene $\mathrm{zu}$ berichten. Craciun et al. beobachteten, dass viele der untersuchten Patienten vor den ersten objektiven Anfallssymptomen kurz innehalten oder aufhören $\mathrm{zu}$ spielen. Die Autoren interpretieren dies als eine Phase möglicher visueller Phänomene, die von den Kindern nicht berichtet werden [7]. Der Mangel an berichteten Auren in Kombination mit falsch lokalisierenden Untersuchungen führt dazu, dass Kinder besonders gefährdet sind, wenn es um die falsche Lokalisation des Anfallsursprungs geht. Insbesondere für pädiatrische Epileptologen ist es daher wichtig, sich der sehr variablen Natur der Anfallssemiologie bei Epilep- sien des posterioren Kortex bewusst $\mathrm{zu}$ sein. Es ist entscheidend, sog. „red flags“ zu erkennen, wie z.B. widersprüchliche Befunde in der prächirurgischen Untersuchung, hohe Anfallsfrequenz, epileptische Spasmen und bereits bestehende visuelle Probleme; 16-30\% der pädiatrischen Patienten haben vorbestehende Gesichtsfelddefekte [3, 5]. Leider ist eine formelle Testung aber oft schwierig und wird als sekundär betrachtet, wenn nicht an den okzipitalen Kortex als Anfallsursprung gedacht wird. Bei Kindern mit einem Verdacht auf eine Beteiligung der posterioren Hirnareale sollte daher eine intrakranielle EEG-Abklärung in Erwägung gezogen. Es wird sich zeigen, ob der vermehrte Einsatz des intrakraniellen EEGs bei Kindern in den letzten Jahren [19] auch bei Kindern mit nichtläsionaler Epilepsie zu mehr posterioren chirurgischen Resektionen führen wird.

\section{Konsequenzen für das intrakranielle EEG}

Prinzipiell gelten für intrakranielle EEGUntersuchungen bei posterioren Epilepsien die gleichen Regeln und Indikationen wie bei allen fokalen Epilepsien. Intrakranielle EEGs können zur Identifikation des Anfallsursprungs in Fällen genutzt werden, in denen keine strukturelle Läsion bekannt ist, im Fall von mehreren Läsionen oder wenn die Grenzen einer Läsion unklar sind. Alternativ sollten sie immer dann in Betracht gezogen werden, wenn die nichtinvasive prächirurgische Diagnostik widersprüchliche Befunde in Bezug auf einen möglichen Anfallsursprung gezeigt hat.

Bisher ist der Nutzen des invasiven EEGs bei posterioren Epilepsien v. a. dann gezeigt worden, wenn ein epileptogenes Areal innerhalb einer großen Läsion nachgewiesen werden sollte oder wenn eine Läsion in dieser Region gesehen wurde, die nicht zu einer temporalen oder frontalen Anfallssemiologie passte 
[2]. Die Abb. 1 fasst einen typischen Fall zusammen, bei dem die Anfallssemiologie und Propagation erst durch invasive Ableitungen verstanden wurde.

Ein weiterer Grund für die Implantationen bei Patienten mit einer Epilepsie des posterioren Kortex ist die funktionelle Stimulation mit dem Ziel, visuelle, sensorische oder sprachliche Funktionen $\mathrm{zu}$ erhalten. In diesen Situationen ist es sehr wichtig, sich der bereits bestehenden Defizite bewusst zu sein. Wenn es um visuelle Funktionen geht, sind Stimulationsstudien vermutlich weniger hilfreich als bei anderen Funktionen. Die Lokalisation der primären visuellen Funktion ändert sich auch bei Patienten mit langjährigen frühen Epilepsien nicht, und dasselbe gilt für die meisten sensorischen Bereiche. Funktionelle Bildgebung und Traktographie könnten daher ein weniger invasiver und dabei zuverlässiger Weg für die Operationsplanung sein [20, 21].

Häufiger als bei anderen Epilepsien werden intrakranielle EEGs bei posterioren Epilepsien dazu genutzt, Propagationswege zu verstehen und zwischen epileptogener und symptomatischer Zone [22] zu unterscheiden. Für diese Aufgabe sind die 3 Implantationsschemata, posterior, zentral-posterior und temporoposterior, hilfreiche Orientierungspunkte, wie von Francione et al. anhand ihrer Patientendaten von 208 Patienten vorgeschlagen [2]. Aus den Ergebnissen der nichtinvasiven präoperativen Untersuchungen ergeben sich somit 3 Implantationsschemata für intrakranielle Elektrodenplatzierungen. Elektroden werden nach einem posterioren Schema implantiert, wenn das epileptische Netzwerk v. a. im oberen Parietallappen, intraparietalen Sulcus, in den angularen und supramarginalen Gyri, dem lingualen Gyrus cuneus, dem pericalcarinen Kortex, dem fusiformen Gyrus und posterioren Cingulum vermutet wird. Das SEEG wird $\mathrm{zu}$ einem temporoposterioren Schema erweitert, wenn zusätzlich zum posterioren Schema die mesial-temporalen Strukturen, die posteriore Insel, die basale Temporalregion, die inferioren, mittleren und oberen temporalen Gyri beteiligt sein könnten.

Ein zentral-posteriores Schema der Implantation wird geplant, wenn zusätz- lich zum posterioren Schema der postzentrale Sulcus, der präzentrale Gyrus, das Operculum, der inferiore frontale Gyrus, der cinguläre Kortex und die posteriore Insula untersucht werden müssen. Diese Implantationsareale sind schematisch in - Abb. 2 dargestellt.

Vergleicht man die vorgeschlagenen Implantationsoptionen von Fancione et al. mit den Propagationswegen von Cracium, dann erlaubt das zentralposteriore Schema eine Untersuchung des dorsalen Propagationswegs, während das temporoposteriore Schema die Ausbreitung nach dem ventralen Weg abdeckt. Interessanterweise kommt es bei Patienten, die nach dem posterioren oder zentral-posterioren Schema implantiert werden, in der Regel eher zu einer fokaleren Resektion und eher zur Anfallsfreiheit als bei Patienten mit einem temporoposterioren Implantationsschema.

In Fällen, in denen das OberflächenEEG diffuse Erregungsmuster zeigte oder nicht lateralisierend war, kann eine bilaterale Abdeckung erforderlich sein [23].

Zusätzlich sollte die intrakranielle Untersuchung auch versuchen, die Ausdehnung der epileptogenen Zone so genau wie möglich abzugrenzen [14]. Dies ist v. a. im Okzipitallappen und im visuellen Kortex von entscheidender Bedeutung, da die postoperativen visuellen Defizite große Auswirkungen auf das tägliche Leben des Patienten haben können. Es ist daher absolut entscheidend, dass die Elektroden sowohl die mesialen als auch die lateralen Aspekte des Okzipitallappens abdecken. Darüber hinaus sollte die Elektrodenplatzierung eine Differenzierung zwischen Arealen oberhalb und unterhalb der calcarinen bzw. parietookzipitalen Fissur ermöglichen [2, 3, 5]. Die vollständige Abdeckung des Okzipitallappens kann eine Herausforderung darstellen. Insbesondere posterior-anterior orientierte Elektroden, die direkt vom Hinterkopf aus eingeführt werden, sollten vermieden werden, da dies für den Patienten unangenehm ist und zum Elektrodenbruch führen kann [23].

Nicht überall ist es möglich, stereotaktische intrakraniellen EEGs durchzuführen. Alternativ können subkortikale EEGs erfolgreich bei der Abgrenzung der epileptogenen Zone helfen [12, 24]. Subkortikale Elektroden können jedoch keine epileptischen Gebiete in tieferen Strukturen des Gehirns lokalisieren. Dies kann eine Herausforderung darstellen, wenn es um die Anfallszone im Okzipitalpol oder in den okzipitalen mesialen Strukturen geht. Ebenso ist die Aussagekraft von subduralen Elektroden limitiert, wenn es darum geht, Propagationswege $\mathrm{zu}$ verstehen [24].

Invasive EEG-Ableitungen sind immer auf die implantierten Hirnareale beschränkt. Es ist daher wichtig, Ausbreitungsmuster in Bereichen, die nicht von intrakranialen Elektroden abgedeckt werden, besser zu verstehen und Fehlinterpretationen zu vermeiden [25]. Die Tatsache, dass Patienten nach intrakraniellen EEG-Untersuchungen bei posterioren Epilepsien nicht häufiger anfallsfrei sind als die Vergleichsgruppe ohne invasives EEG, sollte das Bewusstsein dafür schärfen, dass Epilepsien des posterioren Kortex komplex sind. Es bleibt abzuwarten, ob automatische Analysen des Anfallsursprungs in der Zukunft dabei helfen können, diesen genauer zu definieren und Fehlinterpretationen $\mathrm{zu}$ vermeiden [26].

\section{Schlussfolgerungen}

Epilepsien des posterioren Kortex sind der Kolibri und das Chamäleon der Epilepsien zugleich. Sie sind seltener und präsentieren sich variabler als Epilepsien aus dem Temporal- oder Frontallappen. Aufgrund der starken Vernetzung der posterioren Quadranten und schnellen iktalen Ausbreitung sind sie ein Paradebeispiel dafür, wie die symptomatogene und epileptogene Zone weit voneinander entfernt liegen können. Nicht selten werden posteriore Epilepsien überhaupt nur aufgrund einer strukturellen Läsion erkannt. Gleichzeitig handelt es sich oft um frühe, schwer verlaufende und therapierefraktäre Epilepsien. Der Schlüssel in der Behandlung dieser komplexen Epilepsien liegt daher nicht nur in umfangreicher Diagnostik, sondern auch in einem detaillierten Verständnis involvierter Netzwerke. 


\section{Korrespondenzadresse}

\section{Julia Jacobs, MD, PD}

Hotchkiss Brain Institute and Alberta Children's Hospital Research Institute, University of Calgary

28 Oki Dr NW, T3B 6A8 Calgary, AB, Kanada Julia.jacobs@gmx.de

Funding. Open Access funding enabled and organized by Projekt DEAL.

\section{Einhaltung ethischer Richtlinien}

Interessenkonflikt. S. Steinhoff und J. Jacobs geben an, dass kein Interessenkonflikt besteht.

Für diesen Beitrag wurden von den Autoren keine Studien an Menschen oder Tieren durchgeführt. Für die aufgeführten Studien gelten die jeweils dort angegebenen ethischen Richtlinien.

Open Access. Dieser Artikel wird unter der Creative Commons Namensnennung 4.0 International Lizenz veröffentlicht, welche die Nutzung, Vervielfältigung, Bearbeitung, Verbreitung und Wiedergabe in jeglichem Medium und Format erlaubt, sofern Sie den/die ursprünglichen Autor(en) und die Quelle ordnungsgemäß nennen, einen Link zur Creative Commons Lizenz beifügen und angeben, ob Änderungen vorgenommen wurden.

Die in diesem Artikel enthaltenen Bilder und sonstiges Drittmaterial unterliegen ebenfalls der genannten Creative Commons Lizenz, sofern sich aus der Abbildungslegende nichts anderes ergibt. Sofern das betreffende Material nicht unter der genannten Creative Commons Lizenz steht und die betreffende Handlung nicht nach gesetzlichen Vorschriften erlaubt ist, ist für die oben aufgeführten Weiterverwendungen des Materials die Einwilligung des jeweiligen Rechteinhabers einzuholen.

Weitere Details zur Lizenz entnehmen Sie bitte der Lizenzinformation auf http://creativecommons.org/ licenses/by/4.0/deed.de.

\section{Literatur}

1. Blume WT, Whiting SE, Girvin JP (1991) Epilepsy surgery in the posterior cortex. Ann Neurol 29(6):638-645. https://doi.org/10.1002/ ana.410290611

2. Francione S, Liava A, Cardinale F (2018) SEEG in the posterior cortex epilepsies. In: LhatooSD, Kahane $P_{\text {, }}$ Lüders HO (Hrsg) Invasive studies of the human epileptic brain. Principles and practice. Oxford University Press, Oxford

3. Liava A, Mai R, Tassi Letal (2014) Paediatricepilepsy surgery in the posterior cortex: a study of 62 cases. Epileptic Disord 16(2):141-164. https://doi.org/ 10.1684/epd.2014.0648

4. Ibrahim GM, Fallah A, Albert GW et al (2012) Occipital lobe epilepsy in children: characterization, evaluation and surgical outcomes. Epilepsy Res 99(3):335-345. https://doi.org/10.1016/j. eplepsyres.2011.12.015

5. Ramantani G, Stathi A, Brandt A et al (2017) Posterior cortex epilepsy surgery in childhood and adolescence: predictors of long-term seizure outcome. Epilepsia 58(3):412-419. https://doi. org/10.1111/epi.13654

6. Boesebeck F, Schulz R, May T, Ebner A (2002) Lateralizing semiology predicts the seizure outcome after epilepsy surgery in the posterior cortex. Brain 125(Pt 10):2320-2331. https://doi. org/10.1093/brain/awf236

7. Craciun L, Taussig D, Ferrand-Sorbets S et al (2018) Investigation of paediatric occipital epilepsy using stereo-EEG reveals a better surgical outcome than in adults, especially when the supracalcarine area is affected. Epileptic Disord 20(5):346-363. https:// doi.org/10.1684/epd.2018.1000

8. Olivier A et al (2012) Surgery of occipital lobe epilepsies

9. Rasmussen T (1991) Surgery for central, parietal and occipital epilepsy. Can J Neurol Sci 18(4 Suppl):611-616. https://doi.org/10.1017/ s0317167100032820

10. Naidich TP, Valavanis AG, Kubik S (1995) Anatomic relationships along the low-middle convexity: part I-normal specimens and magnetic resonance imaging. Neurosurgery 36(3):517-532. https:// doi.org/10.1227/00006123-199503000-00011

11. Sierra-Marcos A, Fournier-Del Castillo MC, ÁlvarezLinera J, Budke M, García-Fernández M, PérezJiménez MA (2017) Functional surgery in pediatric drug-resistant posterior cortex epilepsy: electro-clinical findings, cognitive and seizure outcome. Seizure 52:46-52. https://doi.org/10. 1016/j.seizure.2017.09.013

12. Jobst BC, Williamson PD, Thadani VM et al (2010) Intractable occipital lobe epilepsy: clinical characteristics and surgical treatment. Epilepsia 51(11):2334-2337. https://doi.org/10.1111/j. 1528-1167.2010.02673.x

13. Luerding R, Boesebeck F, Ebner A (2004) Cognitive changes after epilepsy surgery in the posterior cortex. J Neurol Neurosurg Psychiatry 75(4):583-587. https://doi.org/10.1136/jnnp.2003.014746

14. Salanova V, Andermann F, Olivier A, Rasmussen $T$, Quesney LF (1992) Occipital lobe epilepsy: electroclinical manifestations, electrocorticography, cortical stimulation and outcome in 42 patients treated between 1930 and 1991. Surgery of occipital lobe epilepsy. Brain 115(Pt 6):1655-1680. https://doi.org/10.1093/brain/115.6.1655

15. Williamson PD, Thadani VM, Darcey TM, Spencer DD, Spencer SS, Mattson RH (1992) Occipital lobe epilepsy: clinical characteristics, seizure spread patterns, and results of surgery. Ann Neurol 31(1):3-13. https://doi.org/10.1002/ana. 410310103

16. Williamson PD, Boon PA, Thadani VM et al (1992) Parietal lobe epilepsy: diagnostic considerations and results of surgery. Ann Neurol 31(2):193-201. https://doi.org/10.1002/ana.410310210

17. Paus T, Collins DL, Evans AC, Leonard G, Pike B, Zijdenbos A (2001) Maturation of white matter in the human brain: a review of magnetic resonance studies. Brain Res Bull 54(3):255-266. https://doi. org/10.1016/s0361-9230(00)00434-2

18. Gerván P, Soltész P, Filep O, Berencsi A, Kovács I (2017) Posterior-anterior brain maturation reflected in perceptual, motor and cognitive performance. Front Psychol 8:674. https://doi.org/ 10.3389/fpsyg.2017.00674

19. Barba C, Cross JH, Braun K et al (2019) Trends in pediatric epilepsy surgery in Europe between 2008 and 2015: country-, center-, and age-specific variation. Epilepsia. https://doi.org/10.1111/epi. 16414
20. Diehl B, Tkach J, Piao Z et al (2010) Diffusion tensor imaging in patients with focal epilepsy due to cortical dysplasia in the temporo-occipital region: electro-clinico-pathological correlations. Epilepsy Res 90(3):178-187. https://doi.org/10. 1016/j.eplepsyres.2010.03.006

21. Collinge S, Prendergast G, Mayers ST et al (2017) Pre-surgical mapping of eloquent cortex for paediatric epilepsy surgery candidates: evidence from a review of advanced functional neuroimaging. Seizure 52:136-146. https://doi. org/10.1016/j.seizure.2017.09.024

22. Rosenow F, Lüders H (2001) Presurgical evaluation of epilepsy. Brain 124(Pt9):1683-1700

23. Chassoux F, Navarro V, Catenoix $H$, Valton L, Vignal J-P (2018) Planning and management of SEEG. Neurophysiol Clin 48(1):25-37. https://doi. org/10.1016/j.neucli.2017.11.007

24. Sakamoto AC, Velasco TR, Gonzalez Martinez J (2018) Subdural EEG in posterior cortex epilepsy. In: Lhatoo SD, Kahane P, Lüders HO (Hrsg) Invasive studies of the human epileptic brain: principles and practice. Oxford Univerity Press, Oxford

25. Ramantani G, Maillard L, Koessler L (2016) Correlation of invasive EEG and scalp EEG. Seizure 41:196-200. https://doi.org/10.1016/j.seizure. 2016.05.018

26. David O, Blauwblomme T, Job A-S et al (2011) Imaging the seizure onset zone with stereo-electroencephalography. Brain 134(10):2898-2911. https://doi.org/10.1093/brain/awr238 